\title{
THE SIZE, DENSITY, AND FORMATION OF THE ORCUS-VANTH SYSTEM IN THE KUIPER BELT
}

\author{
M. E. Brown ${ }^{1}$, D. Ragozzine ${ }^{1,3}$, J. Stansberry ${ }^{2}$, And W. C. Fraser ${ }^{1}$ \\ ${ }^{1}$ Division of Geological and Planetary Sciences, California Institute of Technology, Pasadena, CA 91125, USA; mbrown@ caltech.edu \\ ${ }^{2}$ Steward Observatory, University of Arizona, Tucson, AZ 08544, USA \\ Received 2009 October 25; accepted 2010 April 23; published 2010 May 13
}

\begin{abstract}
The Kuiper Belt object (KBO) Orcus and its satellite Vanth form an unusual system in the Kuiper Belt. While most large KBOs have small satellites in circular orbits and smaller KBOs and their satellites tend to be much closer in size, Orcus sits in between these two regimes. Orcus is among the largest objects known in the Kuiper Belt, but the relative size of Vanth is much larger than that of the tiny satellites of the other large objects. Here, we characterize the physical and orbital characteristics of the Orcus-Vanth system in an attempt to distinguish discuss possible formation scenarios. From Hubble Space Telescope observations, we find that Orcus and Vanth have different visible colors and that Vanth does not share the water ice absorption feature seen in the infrared spectrum of Orcus. We also find that Vanth has a nearly face-on circular orbit with a period of $9.5393 \pm 0.0001$ days and semimajor axis of $8980 \pm 20 \mathrm{~km}$, implying a system mass of $(6.32 \pm 0.01) \times 10^{20} \mathrm{~kg}$ or $3.8 \%$ the mass of dwarf planet Eris. From Spitzer Space Telescope observations, we find that the thermal emission is consistent with a single body with diameter $940 \pm 70 \mathrm{~km}$ and a geometric albedo of $0.28 \pm 0.04$. Assuming equal densities and albedos, this measurement implies sizes of Orcus and Vanth of 900 and $280 \mathrm{~km}$, respectively, and a mass ratio of 33. Assuming a factor of 2 lower albedo for the non-icy Vanth, however, implies sizes of $860 \mathrm{~km}$ and $380 \mathrm{~km}$ and a mass ratio of 12 . The measured density depends on the assumed albedo ratio of the two objects but is approximately $1.5 \pm 0.3 \mathrm{~g} \mathrm{~cm}^{-3}$, midway between typical densities measured for larger and smaller objects. The orbit and mass ratio is consistent with formation from a giant impact and subsequent outward tidal evolution, and even consistent with the system having now achieved a double synchronous state. Because of the large angle between the plane of the heliocentric orbit of Orcus and the plane of the orbit of Vanth, the system can be equally well explained, however, by initial eccentric capture, Kozai cycling to increase the eccentricity and decrease the pericenter of the orbit of Vanth, and subsequent inward tidal evolution. We discuss implications of these formation mechanisms.
\end{abstract}

Key words: astrometry - infrared: planetary systems - Kuiper Belt objects: individual (Orcus) - techniques: high angular resolution

\section{INTRODUCTION}

Orcus is among the brightest known bodies in Kuiper Belt and appears unique in several ways. Its reflectance spectrum shows the deepest water ice absorption of any Kuiper Belt object (KBO) that is not associated with the Haumea collisional family (de Bergh et al. 2005; Trujillo et al. 2005; Barucci et al. 2008). Compositionally, Orcus appears in many ways to be at a transitional size between the moderately common mediumsized KBOs that tend to be spectrally bland (Barkume et al. 2008; Guilbert et al. 2009) and the rare large KBOs that are massive enough to retain volatiles such as methane on their surfaces (Schaller \& Brown 2007). In addition, Orcus has one of the brightest satellites among the large KBOs (Brown 2008), relative to the primary. The large fraction of small satellites around large KBOs led Brown et al. (2006) to suggest that many large KBOs suffered collisions which led to satellite formation. In contrast, the similar brightnesses and large angular momenta of the binary members of other KBO systems have been used to suggest a capture origin for these objects (Goldreich et al. 2002; Noll et al. 2008). Again, Orcus appears to be near the boundary of these two regimes.

Another dichotomy exists between the larger and the smaller KBOs that have satellites. While Eris, Pluto, Quaoar, and Haumea - which all have small presumably collisionally formed satellites-have densities $\sim 2 \mathrm{~g} \mathrm{~cm}^{-3}$ or higher (Brown \&

\footnotetext{
3 Now at Harvard-Smithsonian Center for Astrophysics, Cambridge, MA 02138, USA.
}

Schaller 2007; Buie et al. 2006; Rabinowitz et al. 2006; Fraser \& Brown 2010), smaller Kuiper Belt binaries have densities of $1 \mathrm{~g} \mathrm{~cm}^{-3}$ and even lower (Stansberry et al. 2006; Grundy et al. 2008). Orcus, being intermediate in size between the two populations, could help to shed insight on this apparent bifurcation.

In order to investigate the properties of the Orcus system, we obtained Hubble Space Telescope (HST) observations to determine the orbit, and optical and infrared colors of the components of the system, and Spitzer Space Telescope observations of thermal emission in order to determine the size and thus density of the system.

\section{THE ORBIT OF VANTH}

Vanth, the satellite of Orcus, was discovered in observations from the High Resolution Camera (HRC) of the Advanced Camera for Surveys (ACS) on HST obtained on 2005 November 13. Follow-up observations were obtained a year later in order to determine the orbit of the system. In each HST orbit, eight exposures were obtained in the F606W filter with $275 \mathrm{~s}$ exposures.

In order to determine accurate astrometric positions of the satellite relative to Orcus, we performed detailed point-spread function (PSF) fitting of the system. A 5 times oversampled theoretical PSF was constructed for the approximate pixel location of Orcus on the HRC image using the HST PSF modeling software TinyTim, and a least-squares fit was performed optimizing the sub-pixel centers of Orcus and the satellite, the total 


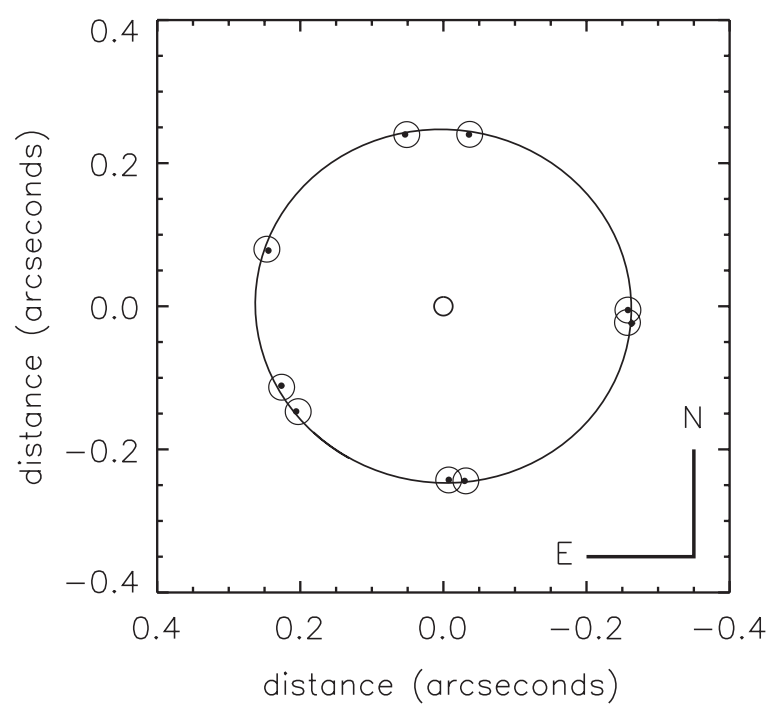

Figure 1. Orbit of Vanth. The data points are shown with their error bars, while predicted positions from the best-fit models are shown as large circles. Orcus is shown approximately to scale at the center.

Table 1

Separation of Orcus and its Satellite

\begin{tabular}{cccc}
\hline \hline $\begin{array}{c}\text { Date } \\
(\mathrm{UT})\end{array}$ & $\begin{array}{c}\text { R.A. Offset } \\
(\mathrm{mas})\end{array}$ & $\begin{array}{c}\text { Decl. Offset } \\
(\mathrm{mas})\end{array}$ & Instrument \\
\hline 2453687.664 & $206 \pm 3$ & $-147 \pm 3$ & ACS \\
2454040.369 & $226 \pm 4$ & $-111 \pm 3$ & ACS \\
2454044.366 & $-258 \pm 1$ & $-005 \pm 1$ & ACS \\
2454051.579 & $-006 \pm 1$ & $-243 \pm 1$ & ACS \\
2454056.089 & $-036 \pm 1$ & $240 \pm 1$ & ACS \\
2454066.146 & $053 \pm 2$ & $240 \pm 1$ & ACS \\
2454080.336 & $-030 \pm 1$ & $-244 \pm 1$ & ACS \\
2454416.293 & $-263 \pm 2$ & $-024 \pm 5$ & NICMOS \\
2454439.780 & $245 \pm 3$ & $078 \pm 4$ & WFPC2 \\
\hline
\end{tabular}

flux of Orcus and the satellite, and the magnitude of the sky background. The uncertainties for each observation are determined from the scatter within a single HST orbit. In some cases, it appears that individual observations within an orbit detect consistent motion on minutes-long timescales; nonetheless, we assume that all deviation within one orbit is due to measurement error. Observations of the Orcus system were also obtained with the Wide Field Planetary Camera 2 (WFPC2) on HST as well as NICMOS, the infrared camera. Astrometric positions of the satellite were determined from these observations identically, using appropriate PSFs for each camera and filter. All astrometric observations are shown in Figure 1 and Table 1.

From the astrometric observations, it appears that the Orcus satellite is close to being pole-on and is in an essentially circular orbit.

We determine the orbit using a Powell $\chi^{2}$ minimization scheme to find the optimal orbital parameters. We first attempt to fit a purely circular orbit in which the five free parameters are semimajor axis, orbital period, inclination, longitude of the ascending node, and mean anomaly. The best fit has a $\chi^{2}$ value of 9.5 , or a reduced $\chi^{2}$ for 13 degrees of freedom (nine sets of $x, y$ coordinates minus five orbital parameters) of 0.73 , indicating an excellent fit to the model (and perhaps slightly over estimated error bars). Expanding the model to allow an eccentric fit gives a best-fit eccentricity of 0.002 and a slightly lower reduced $\chi^{2}$ of 0.52 . Given the excellent fit to the circular orbit and the only
Table 2

Orbital Parameters ${ }^{\mathrm{a}}$

\begin{tabular}{lcc}
\hline \hline \multicolumn{1}{c}{ Parameter } & Solution 1 & Solution 2 \\
\hline Reduced $\chi^{2}$ & 0.73 & 0.75 \\
Semimajor axis & $8980 \pm 23$ & $8985 \pm 24 \mathrm{~km}$ \\
Period & $9.5393 \pm 0.0001$ & $9.5392 \pm 0.0001$ days \\
Inclination & $90.2 \pm 0.6$ & $305.8 \pm 0.6$ \\
Longitude of ascending node & $50.0 \pm 0.6$ & $249.4 \pm 0.4$ \\
Mean anomaly & $143.1 \pm 0.02$ & $316.6 \pm 0.2$ \\
Epoch (defined) & JD 2454439.780 & \\
\hline
\end{tabular}

Note. ${ }^{\text {a }}$ Relative to J2000 ecliptic.

slight improvement when eccentricity is allowed, we conclude that no evidence for a non-circular orbit is found.

To determine uncertainties in the individual parameters, we perform 1000 iterations of circular orbit fit optimization where we add Gaussian noise with $\sigma$ equal to the measurement uncertainties of the position measurements and solve for new orbital parameters. We define the $1 \sigma$ uncertainties on the parameters to be the range containing the central $68 \%$ of the data. To estimate an upper limit to the eccentricity, we perform an additional 1000 iterations allowing a fully eccentric fit and take the $1 \sigma$ upper limit to eccentricity to be the point higher than $68 \%$ of the data. We find an upper limit of 0.0036 to the eccentricity of the orbit. Table 2 gives the ecliptic orbital elements of the satellite orbit.

The circular orbit gives a system mass of $(6.32 \pm 0.05) \times$ $10^{20} \mathrm{~kg}$, or about $3.8 \%$ the mass of the largest known dwarf planet, Eris.

As with most KBO satellite orbits, astrometric data have not been obtained over a long enough time interval to break the geometric plane-of-sky degeneracy, thus two similar solutions can be found. The second solution has a reduced $\chi^{2}$ of 0.75 compared to 0.73 for the nominal solution, indicating that both are excellent fits. The two solutions give nearly identical orbital distances and periods and thus identical masses. Both solutions and their uncertainties are shown in Table 2. For both solutions, the orbit of Vanth is highly inclined compared to the orbit of Orcus, with an angle between the pole vectors of $73^{\circ}$ or $109^{\circ}$, and nearly pole-on to the Sun, with an angle between the pole vector and the Sun-Orcus line of $29^{\circ}$ or $31^{\circ}$.

\section{VISIBLE PHOTOMETRY OF ORCUS AND ITS SATELLITE}

The unusual spectral properties of the satellites of Haumea (Barkume et al. 2006; Fraser \& Brown 2009) have been used to argue that these satellites cannot have been captured from the typical Kuiper Belt population. Indeed, the deep water ice absorption features seen on the satellites of Haumea have been argued to be a consequence of formation by a giant impact on a previously differentiated body. Similar deep water ice absorption features on Charon (Brown \& Calvin 2000) suggest that such spectral features could be a general signature of formation in a giant impact.

An examination of the spectral properties of the satellite of Orcus could likewise help to reveal its origin. The satellite is, however, significantly closer to the primary than is the outer satellite of Haumea; thus, to determine the surface composition of the satellite, ground-based spectroscopy is virtually impossible. We resort, instead, to HST spectrophotometry as a proxy for spectroscopy. Fraser \& Brown (2009) demonstrated that such spectrophotometry could detect the presence of deep water ice 
Table 3

Photometry of Orcus and Vanth

\begin{tabular}{ccccc}
\hline \hline Date & Instrument & Filter/Band & Orcus & Vanth \\
\hline 2454439.280 & HST/WFPC2 & F606W & $19.186 \pm 0.006$ & $21.73 \pm 0.01$ \\
& & F814W & $18.62 \pm 0.02$ & $20.90 \pm 0.02$ \\
& Johnson $V$ & $19.36 \pm 0.05$ & $21.97 \pm 0.05$ \\
& Cousins $I$ & $18.63 \pm 0.05$ & $20.94 \pm 0.05$ \\
2454415.793 HST/NICMOS & F110W & $20.64 \pm 0.04$ & $23.3 \pm 0.3$ \\
& F160W & $21.65 \pm 0.03$ & $24.0 \pm 0.02$ \\
& Spitzer/MIPS & 24 & $0.378 \pm 0.3$ mJy & (Unresolved) \\
& 70 & $25.0 \pm 2.4$ mJy (Unresolved)
\end{tabular}

absorption on the satellites of Haumea. HST can likewise easily resolve the Orcus system and provide separate photometry of the two components.

Observations of the optical and infrared colors of Orcus and its satellite were obtained with WFPC2 and NICMOS on HST on 2007 December 5 and 2008 November 11, respectively. Photometry was performed using the same PSF-fitting as described above for astrometric fitting of the points, and the results are shown in Table 3. Uncertainties are obtained from the scatter of the individual measurements.

Colors of Orcus and its satellite are shown in Figure 2 for both the native instrument magnitude system and an approximate conversion to Johnson $V$-Cousins $I$. For the conversion, we construct a series of linearly reddened solar spectra and use the IRAF package synphot to calculate both sets of colors. We also use the best-fit reddened spectrum to approximately convert the F606W magnitudes to Johnson $V$ and F814W magnitudes to Cousins $I$ for both objects. While the color conversion should be quite accurate, we assume that the accuracy of the single band conversion is no better than 5\%. With the derived $V$-band magnitudes of Orcus and Vanth of $19.36 \pm 0.05$ and $21.97 \pm$ 0.05 and assuming the phase function for Orcus measured by Sheppard (2007), we obtain absolute magnitudes of Orcus and Vanth of $H_{V}=2.27 \pm 0.05$ and $4.88 \pm 0.05$, respectively. The combined absolute magnitude of $H_{V}=2.17 \pm 0.05$ agrees well with the measured value in $R$ band of $1.81 \pm 0.005$ (Sheppard 2007) and the solar color of $V-R=0.37$.

For a comparison, similarly obtained colors of Haumea and its satellites are also shown in Figure 2. While Haumea and its satellites show the characteristic neutral color and clear signature of deep water ice absorption at $1.6 \mu \mathrm{m}$, the satellite of Orcus has a moderately red spectral slope at optical wavelengths and appears flat across the infrared, spectral characteristics that are typical in the Kuiper Belt (Barkume et al. 2008; Guilbert et al. 2009).

Unlike the satellites of Haumea, the visible-infrared spectrum of the satellite of Orcus does not argue against a capture origin. Unfortunately, without a clearer understanding of the origin of the deep water ice absorption features on the satellites of Haumea and on Charon, we cannot conclusively argue that the lack of such spectral features on the satellite of Orcus is a compelling argument that the satellite must have been captured.

Regardless of the origin of the satellite, the difference between the visible colors of Orcus and its satellite is a stark contrast between the very strong correlation between primary and satellite colors observed by Benecchi et al. (2009). Indeed, no other measured satellite-primary pair appears so discrepant. Benecchi et al. argue that the satellite-primary color correlation suggests that KBO colors are primordial and a function of formation location. If satellite capture only occurs within a limited zone then satellites and primaries should have similar

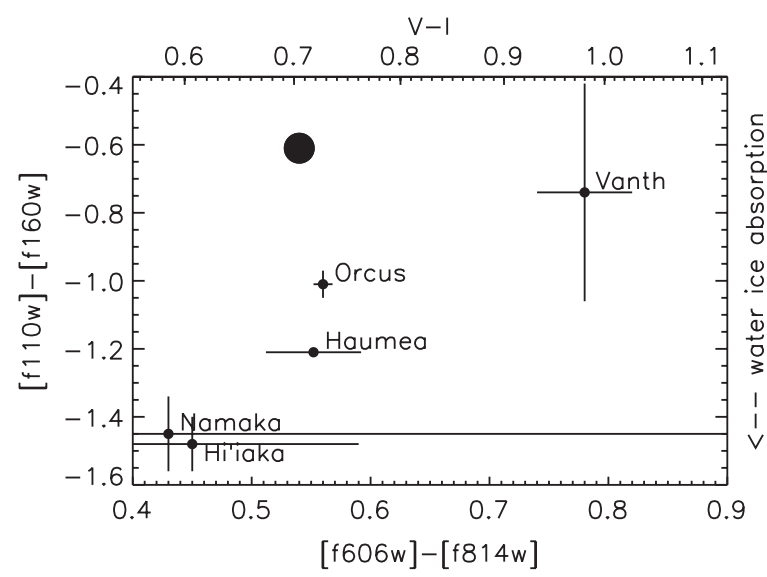

Figure 2. Visible and infrared photometry of the Orcus and Haumea systems While the visible and infrared colors of Haumea and its satellites Hi' iaka and Namaka are all consistent with having deep water ice spectral features, the colors of Orcus and Vanth are not. Orcus is known to have moderate water ice absorption, which can be seen in the moderate blue [f110W]-[f160W] colors. The large circle shows solar colors in these filter systems.

colors. The discrepant colors of Orcus and Vanth could be used to argue, therefore, that Vanth is not a captured satellite. A compelling argument could be made, however, that larger KBOs (at least those not large enough to maintain methane) are preferentially bluer, like Orcus, thus the color of Orcus could reflect evolution rather than origin, while the color of Vanth would be closer to the colors of the objects of the region in which Orcus and Vanth formed. While no statistically significant evidence for such a size-color correlation has been reported (Doressoundiram et al. 2008), this lack of significance reflects as much the very small numbers of large objects as it does the observations. We are thus reluctant to make a conclusion based on the different optical colors of Orcus and Vanth.

The different colors certainly indicate different surface types and suggest the possibility that Orcus and Vanth could have quite different albedos.

\section{THE SIZE AND DENSITY OF ORCUS}

To further characterize the properties of the Orcus system, we obtained far-IR observations of the thermal emission from Orcus using the Multiband Imaging Photometer (MIPS) instrument on the Spitzer Space Telescope. These, and similar observations of other trans-Neptunian objects, were described in Stansberry et al. (2008). Here, we briefly re-summarize the data analysis and photometric methods.

Data were taken in the MIPS $24 \mu \mathrm{m}$ and $70 \mu \mathrm{m}$ channels using the photometry observing template. Observations were acquired on 2007 May 30 and 31. Data were processed through the MIPS instrument team analysis pipeline, resulting in absolutely calibrated images. Between the two observations, Orcus moved $35^{\prime \prime}$. We took advantage of that motion to subtract off background sources in the images before performing photometry. This sky subtraction provided a factor of $\simeq 2$ improvement in signal-to-noise ratio in both bands. Finally, we coadded the sky-subtracted images from the two epochs in the comoving frame, producing a single final image in each MIPS band.

Photometry was measured using circular apertures with radii 5" and 14".8 at 24 and $70 \mu \mathrm{m}$, respectively, and appropriate aperture corrections applied to the result (Gordon et al. 2007; Engelbracht et al. 2007). 


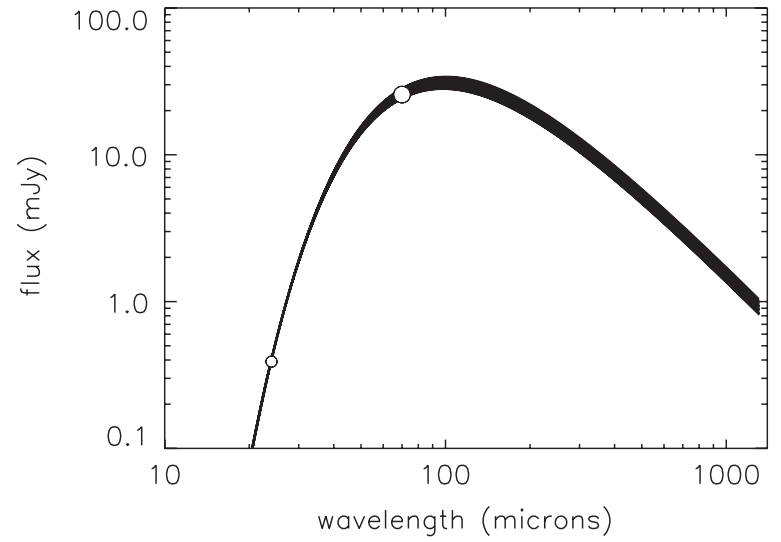

Figure 3. Thermal models for Orcus. The 24 and $70 \mu \mathrm{m}$ points from Spitzer are shown with $1 \sigma$ error bars. The suite of models which provide a $1 \sigma$ or better fit to these two data points is shown.

We calculate flux values of $0.378 \pm 0.03 \mathrm{mJy}$ at $24 \mu \mathrm{m}$ and $25.0 \pm 1.4 \mathrm{mJy}$ at $70 \mu \mathrm{m}$, where the fluxes are from the average of four measurements and the uncertainties are determined from the scatter in the observations. Including the $4 \%$ and $8 \%$ calibration uncertainties at 24 and $70 \mu \mathrm{m}$, our final photometric measurements are $0.378 \pm 0.03$ and $25.0 \pm 2.4 \mathrm{mJy}$ at 24 and $70 \mu \mathrm{m}$, respectively.

We approach modeling of thermal emission using the same suite of thermal models (Lebofsky \& Spencer 1989) with a different philosophy than has been done previously (Stansberry et al. 2008; Brucker et al. 2009). Results are similar, but our analysis has the potential to give more physical insight into the parameters used.

In the standard thermal model (STM), the surface temperature at each location on the body is assumed to be in instantaneous thermal equilibrium between incoming sunlight and outgoing thermal emission. The total thermal emission of the body can be found by integrating the emission over the entire surface. The other main end-member used in modeling is the isothermal latitude model (ILM) in which each surface element is in radiative equilibrium with the average sunlight that is seen over the course of a rotation, leading to a surface temperature which is a function of latitude.

Even for the lowest plausible values of thermal inertia, objects at the $\sim 40-50 \mathrm{~K}$ temperatures in the Kuiper Belt radiate so slowly that their surfaces do not have time to radiate significant heat over typical Kuiper Belt rotation periods. A simple order-ofmagnitude demonstration of this fact can be found by calculating the total temperature change over a rotation in a volume one thermal skin depth deep. For any plausible thermal parameters, the temperature change is only a fraction of a degree. We thus conclude that the ILM is the only physically plausible models. The ILM and STM are identical when the rotation pole points directly at the Sun.

The other main parameters used in these thermal models are $q$, the phase integral, which relates the optical albedo to the Bond albedo, and $\eta$, the beaming factor, which is a simple correction to the total amount of energy radiated in the sunward direction, usually assumed to be caused by surface roughness, but which can be taken as a generic correction factor to the assumed temperature distribution. For asteroids of known sizes, Lebofsky et al. (1986) found $\eta$ to be approximately 0.75 , a correction which agrees well with measurements of icy objects in the outer solar system objects (Brown et al. 1982a, 1982b). As no beaming factor has been measured for any object in the

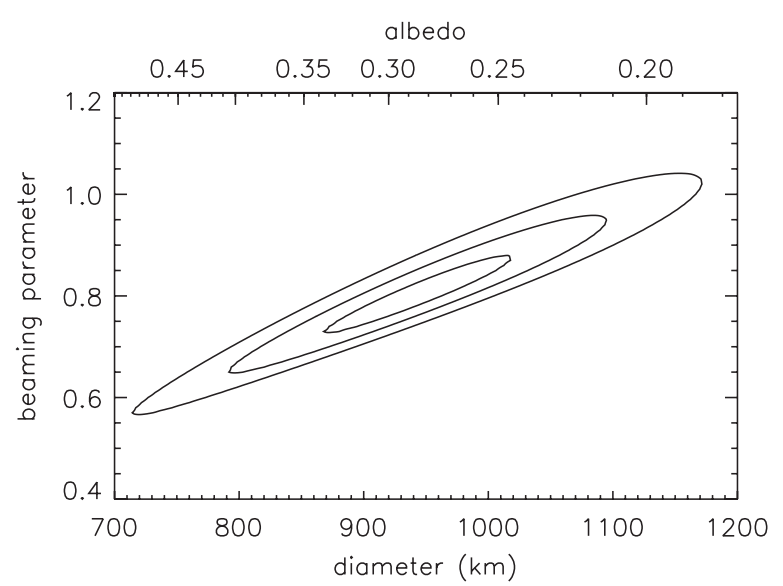

Figure 4. Error contours for thermal model fits. Thermal fluxes from a large grid of possible models with variable albedo, phase integral, and albedo were calculated. The best fits have beaming parameters within the range of expected values.

Kuiper Belt, we allow this factor to be a free parameter in our models, though we keep in mind that a value of approximately 0.75 appears to be widely applicable in the solar system.

The Bond albedos or phase integrals of KBOs have also never been measured, but we find that the phase integrals of the large icy satellites of Uranus, whose infrared spectra are quite similar to the infrared spectrum of Orcus, all have measured phase integrals between 0.45 and 0.65 (Buratti et al. 1990). We use this as a representative range for the phase integral of Orcus with the caveat that if the albedo of Orcus turns out to be significantly different from those of the Uranian satellites, we should revisit the appropriateness of the surface analogy.

Finally, in our models, we fix the pole position of Orcus to be aligned with the derived pole positions of the orbit of Vanth. If Vanth were created in a collision and tidally evolved outward, the poles would be expected to be aligned. For other formation scenarios, alignment of the rotation and satellite pole positions is still a likely outcome, but not required (see below).

With these choices of parameters, we calculate a suite of thermal models encompassing a full range of albedos, phase integrals, and beaming parameters. Each model is scaled to a size to give it the total $V$-band absolute magnitude of $2.17 \pm$ 0.06 from the WFPC2 data, then fluxes in the MIPS 24 and $70 \mu \mathrm{m}$ bands are calculated. Rather than attempting thermal color corrections, as has been done by all previous KBO thermal modelers, we instead directly integrate the flux in the full bands using the published 24 and $70 \mu \mathrm{m}$ filter functions so that we can directly compare our measured fluxes to the models.

To assess the quality of the fit of any model, we define

$$
\begin{aligned}
\sigma=[ & \left(F 24_{\text {model }}-F 24_{\text {measured }}\right)^{2} / \sigma_{24}^{2} \\
& \left.+\left(F 70_{\text {model }}-F 70_{\text {measured }}\right)^{2} / \sigma_{70}^{2}\right],
\end{aligned}
$$

where $F 24_{\text {model }}$ and $F 24_{\text {measured }}$ are the $24 \mu \mathrm{m}$ modeled and measured fluxes, $\sigma_{24}$ is the uncertainty in the $24 \mu \mathrm{m}$ measured flux, and $F 70_{\text {measured }} F 70_{\text {modeled }}$, and $\sigma_{70}$ are identical parameters at $70 \mu \mathrm{m}$.

Figure 3 shows the 24 and $70 \mu \mathrm{m}$ fluxes as well as the suite of thermal models that fall within $1 \sigma$ of the measurements. Figure 4 shows a contour plot of $\sigma$ values as a function of diameter, albedo, and beaming parameter. The best fit and full $1 \sigma$ range of uncertainty to the diameter and the geometric albedo are $940 \pm 70 \mathrm{~km}$ and $0.28 \pm 0.04$, respectively. The $1 \sigma$ range 
of acceptable beaming parameters varies from 0.70 to 0.88 . This range encompasses the expected value of approximately 0.75 previously found to be appropriate for asteroids and icy satellites, giving confidence to the results. The derived albedo is also similar to that of the icy satellites of Uranus that were used for phase integral analogs, further bolstering confidence.

The total thermal flux also includes the flux from Vanth. In the F606W filter, Vanth is $2.54 \pm 0.01$ magnitudes fainter than Orcus, suggesting a diameter 3.2 times smaller for similar albedos. Given the moderately high albedo of Orcus, however, and the dissimilar colors of Orcus and Vanth, we entertain the possibility that Vanth could have an albedo as much as a factor of 2 lower than Orcus. For similar albedos, the combined sizes which yield an equivalent surface area as that of a $940 \mathrm{~km}$ single object are $900 \mathrm{~km}$ and $280 \mathrm{~km}$ for Orcus and Vanth, respectively. Assuming similar densities (which, again, might not be the case for this pair), the mass ratio is 33. If Vanth has an albedo half of that of Orcus, the diameters would instead be $860 \mathrm{~km}$ and $380 \mathrm{~km}$ with an equal-density mass ratio of only 12 . (Owing to the wide range of possible size and mass ratios, we have not explicitly recalculated thermal models for pairs of dissimilar objects, so true best-fit model sizes will differ slightly.)

The single-body size and mass of Orcus combine to give a density of $1.45 \pm 0.3 \mathrm{~g} \mathrm{~cm}^{-3}$, though partitioning the thermal emission to two bodies raises the best-fit density to $1.6 \mathrm{~g} \mathrm{~cm}^{-3}$ for the identical albedo assumption and to $1.7 \mathrm{~g} \mathrm{~cm}^{-3}$ for the low albedo for Vanth. Regardless of the partitioning, Orcus and Vanth appear to have a density in the intermediate range between the high- and low-density clumps of KBOs.

\section{THE ORIGIN OF THE ORCUS SATELLITE SYSTEM}

\subsection{Collision Plus Tidal Evolution}

The close circular orbit of Vanth demands circularization through tidal evolution. Such tidal evolution would be expected from a collisionally formed satellite that evolved outward.

In this scenario, Vanth reaccumulated from post-giant impact debris in an orbit just beyond the Roche limit, and the postcollision Orcus was rapidly rotating from the collision. Tidal torques then transferred angular momentum from Orcus' spin to the satellite orbit and expanded its orbit outward. Eventually, the orbit of Vanth will expand until the spin rate of both Orcus and Vanth reaches the orbital frequency and Orcus and Vanth attain the double synchronous state, as has occurred for Pluto and Charon.

If Orcus and Vanth are currently in the double synchronous state, we can put limits on their mass ratio. Assuming that both objects rotate with the 9.53 day period of the orbit, the total angular momentum of the current system matches a system where the primary is rotating at breakup and the secondary orbits at the Roche radius if the mass ratio is approximately 8 , smaller than the likely allowed mass ratio. If the post-impact Orcus was not rapidly rotating and had a spin period more like the $\sim 10 \mathrm{hr}$ periods of typical KBOs, however, the orbit would be doubly synchronous with a mass ratio of 32 . The uncertainty in the range of end states allowed after a giant impact precludes using the current orbital configuration as a measurement of the mass ratio.

We can estimate the timescale to tidally evolve to the current orbital distance as

$$
t=\frac{2}{39}\left(\frac{a}{r}\right)^{5} \frac{m_{p}}{m_{s}} \frac{1}{n} \frac{Q}{k_{2}},
$$

where $a$ is the satellite semimajor axis, $r$ is the radius of the primary, $m_{p} / m_{s}$ is the primary to secondary mass ratio, $n$ is the orbital frequency, and $Q$ and $k_{2}$ are the tidal dissipation factor and Love number, respectively (Murray \& Dermott 2000). For a mass ratio of 12 , this timescale becomes $9000 \mathrm{yr} \times\left(Q / k_{2}\right)$ or about $24,000 \mathrm{yr} \times\left(Q / k_{2}\right)$ for a mass ratio of 32 . Using order-of-magnitude estimates of $Q=100$ and $k_{2}=0.005$ from Murray \& Dermott (2000), we estimate a synchronization timescale of 150-400 Myr for the system. Even within the large uncertainties, this estimate is comfortably smaller than the age of the solar system, consistent with the idea that the system evolved to a synchronous state and then stopped, and suggesting a post-impact rotation speed smaller than breakup. The eccentricity damping timescale is even faster, so that a circular orbit is expected due to tidal dissipation.

Is there any evidence for a double synchronous state? Unfortunately, if the spin axis of Orcus is aligned with the orbit-normal (as expected for a tidally evolved system), the pole-on nature of the system implies that observing rotational photometric variability from Orcus is nearly impossible. Alternatively, a robust detection of a spin rate faster than the 9.5 day period of the Vanth orbit would conclusively demonstrate that this state has not been achieved.

\subsection{Inward Tidal Evolution}

While outward tidal evolution can reproduce the current state of the Orcus-Vanth system, tidal evolution can also proceed inward from a more extended orbit if Vanth were captured rather than formed in a collision. For such an evolution to produce the currently observed system, however, the capture would have had to start with a highly unusual orbit. Because tidal evolution conserves the total angular momentum of the system, and because the total angular momentum of a system with a more extended orbit is dominated by the orbital angular momentum, this orbital angular momentum must stay approximately constant or increase. Thus, the quantity $a\left(1-e^{2}\right)=a(1-e)(1+e)$ must be conserved, so the initial pericenter of the orbit $q_{\text {init }}=$ $a_{\text {init }}\left(1-e_{\text {init }}\right)$ must be smaller than the current pericenter as long as $a$ has decreased. While no general theoretical understanding of the initial $a$ and $e$ distributions of captured Kuiper Belt satellites exists, invoking an initial orbit with an initial pericenter even closer than the 19 primary radii of the current orbit appears implausible. We thus reject this scenario as possible formation mechanism for the Orcus-Vanth system.

\subsection{Kozai Cycles with Tidal Friction}

While an initially low pericenter orbit appears implausible, secular perturbations due to the Sun can sometimes create large eccentricities and low pericenters through Kozai oscillation (Perets \& Naoz 2009). Kozai oscillations or Kozai cycles are large variations in eccentricity and inclination which can occur when the relative inclination between the heliocentric orbit and the binary mutual orbit exceeds a critical value (typically $40^{\circ}<i<140^{\circ}$ ). These oscillations cause the eccentricity and inclination of the KBO binary to exchange on long timescales while keeping $\sqrt{1-e^{2}} \cos i$ constant. The Orcus-Vanth system has a mutual inclination of either $73^{\circ}$ or $109^{\circ}$ putting it within the range where Kozai oscillations could have effected it.

In the Kozai cycles scenario, Vanth is captured by Orcus on a typical high semimajor axis moderate eccentricity orbit which happens to have a high mutual inclination. This high mutual inclination allows Kozai cycling to trade eccentricity and 
inclination, leading to phases at which the orbit is extremely eccentric but the semimajor axis remains unchanged. The pericenter of the satellite orbit can thus drop down into the range where, like discussed above, significant tidal evolution can finally occur at which point the semimajor axis of the orbit can shrink dramatically.

In Ragozzine (2009), we present detailed models of this evolutionary path showing that Kozai cycles with tidal friction can produce orbital parameters similar to the Orcus-Vanth system from a variety of starting conditions.

\section{CONCLUSIONS}

Two dynamical scenarios appear equally plausible for explaining the formation of the Orcus-Vanth binary. The orbital characteristics of Vanth are consistent with formation in a subcatastrophic giant impact such as that is thought to have formed the Pluto-Charon system (Canup 2005). Indeed, with Orcus in a nearly identical heliocentric orbit as Pluto suggesting an analogy between Pluto-Charon formation and Orcus-Vanth formation is pleasing.

The Orcus-Vanth system could be equally well explained, however, by capture, Kozai cycling, and subsequent tidal evolution (which cannot explain Pluto-Charon due to the presence of additional circular coplanar satellites in that system). While the 2.54 mag brightness difference between Orcus and Vanth is moderately extreme compared to other binaries which are thought to have formed by capture (Noll et al. 2008), if Vanth has an albedo lower than Orcus by a factor of 2 , as seems plausible, the equivalent magnitude difference is only 1.8 , closer to values observed in typical systems.

We have hypothesized that perhaps all collisionally formed satellites should share similar spectral characteristics, with the neutral colors and deep water ice absorptions found in the Haumea system and on Charon. While the presence of these unusual spectral features on Vanth would have been significant evidence for a collisional origin, it is difficult to argue that their absence rules out collisional origin. Without a significantly more detailed understanding of the physics of icy body collisions and subsequent surface evolution, we are unwilling to assert that collision could not have formed a satellite with a surface like Vanth.

Finally, we have noted that the largest KBOs with small presumably collisionally formed satellites all have densities higher than those of the smaller satellites. The uncertainty in the density measured for Orcus, unfortunately, perfectly straddles the range between those low-density captured objects and higher-density objects like Pluto and Charon (though it is inconsistent with the extremely high densities of objects like Haumea and Quaoar).

The origin of the Orcus-Vanth binary remains uncertain. Future observations which may help to constrain this origin include more precise measurements of the size of Orcus, to determine whether Orcus fits into the high or into the low densities found in the Kuiper Belt (or elsewhere), and a deep search for additional coplanar satellites, analogous to Nix and Hydra, which would firmly rule out a capture origin.

This research has been supported by grants from STScI and SSC and through the NASA Earth and Space Science Fellowship program.

\section{REFERENCES}

Barkume, K. M., Brown, M. E., \& Schaller, E. L. 2006, ApJ, 640, L87

Barkume, K. M., Brown, M. E., \& Schaller, E. L. 2008, AJ, 135, 55

Barucci, M. A., et al. 2008, A\&A, 479, L13

Benecchi, S. D., Noll, K. S., Grundy, W. M., Buie, M. W., Stephens, D. C., \& Levison, H. F. 2009, Icarus, 200, 292

Brown, M. E. 2008, in The Solar System Beyond Neptune, ed. M. A. Barucci, H Boehnhardt, D. P. Cruikshank, \& A. Morbidelli (Tucson, AZ: Univ. Arizona Press), 335

Brown, M. E., \& Calvin, W. M. 2000, Science, 287, 107

Brown, M. E., \& Schaller, E. L. 2007, Science, 316, 1585

Brown, M. E., et al. 2006, ApJ, 693, L43

Brown, R. H., Cruikshank, D. P., \& Morrison, D. 1982a, Nature, 300, 423

Brown, R. H., Morrison, D., Telesco, C. M., \& Brunk, W. E. 1982b, Icarus, 52, 188

Brucker, M. J., Grundy, W. M., Stansberry, J. A., Spencer, J. R., Sheppard, S. S., Chiang, E. I., \& Buie, M. W. 2009, Icarus, 201, 284

Buie, M. W., Grundy, W. M., Young, E. F., Young, L. A., \& Stern, S. A. 2006, AJ, 132,290

Buratti, B., Wong, F., \& Mosher, J. 1990, Icarus, 84, 203

Canup, R. M. 2005, Science, 307, 546

de Bergh, C., Delsanti, A., Tozzi, G. P., Dotto, E., Doressoundiram, A., \& Barucci, M. A. 2005, A\&A, 437, 1115

Doressoundiram, A., Boehnhardt, H., Tegler, S. C., \& Trujillo, C. 2008, in The Solar System Beyond Neptune, ed. M. A. Barucci et al. (Tucson, AZ: Univ. Arizona Press), 91

Engelbracht, C. W., et al. 2007, PASP, 119, 994

Fraser, W. C., \& Brown, M. E. 2009, ApJ, 695, L1

Fraser, W. C., \& Brown, M. E. 2010, ApJ, 714, 1547

Goldreich, P., Lithwick, Y., \& Sari, R. 2002, Nature, 420, 643

Gordon, K. D., et al. 2007, PASP, 119, 1019

Grundy, W. M., et al. 2008, Icarus, 197, 260

Guilbert, A., Alvarez-Candal, A., Merlin, F., Barucci, M. A., Dumas, C., de Bergh, C., \& Delsanti, A. 2009, Icarus, 201, 272

Lebofsky, L. A., \& Spencer, J. R. 1989, in Asteroids II, ed. T. Gehrels \& M. S. Matthews (Tucson, AZ: Univ. Arizona Press), 128

Lebofsky, L. A., et al. 1986, Icarus, 68, 239

Murray, C. D., \& Dermott, S. F. 2000, Solar System Dynamics (Cambridge: Cambridge Univ. Press)

Noll, K. S., Grundy, W. M., Chiang, E. I., Margot, J.-L., \& Kern, S. D. 2008, in The Solar System Beyond Neptune, ed. M. A. Barucci et al. (Tucson, AZ: Univ. Arizona Press), 345

Perets, H. B., \& Naoz, S. 2009, ApJ, 699, L17

Rabinowitz, D. L., Barkume, K., Brown, M. E., Roe, H., Schwartz, M., Tourtellotte, S., \& Trujillo, C. 2006, ApJ, 639, 1238

Ragozzine, D. A. 2009, PhD Thesis, Caltech (http://resolver.caltech.edu/ CaltechETD:etd-05282009-164537)

Schaller, E. L., \& Brown, M. E. 2007, ApJ, 659, L61

Sheppard, S. S. 2007, AJ, 134, 787

Stansberry, J., Grundy, W., Brown, M., Cruikshank, D., Spencer, J., Trilling, D., \& Margot, J.-L. 2008, in The Solar System Beyond Neptune, ed. M. A. Barucci et al. (Tucson, AZ: Univ. Arizona Press), 161

Stansberry, J. A., Grundy, W. M., Margot, J. L., Cruikshank, D. P., Emery, J. P., Rieke, G. H., \& Trilling, D. E. 2006, ApJ, 643, 556

Trujillo, C. A., Brown, M. E., Rabinowitz, D. L., \& Geballe, T. R. 2005, ApJ, 627,1057 